\title{
The Most Common Damaged Primary Teeth in Children
}

\author{
Mohammed Karimi DMD* \\ Sepideh Dental Clinic, Department of Pediatric Dentistry, Iran
}

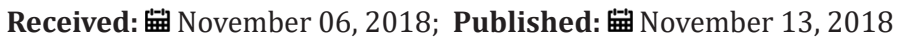

*Corresponding author: Mohammed Karimi, Sepideh Dental Clinic, Pediatric Department, Iran

\section{Editorial}

Damage to the teeth of a young child can leave serious and prolonged periods of time, leading to a change in color, deformity or possible loss of it. The psychological impact of such damage can be very wide. Therefore, the pediatric dentist should have the following characteristics such as: having the knowledge of the treatment of the traumatized tooth and being available to provide the necessary treatment and services at any time of the day. The most common teeth that are damaged in the primary dental system are maxillary incisors. Primary molars are rarely damaged and are often damaged by indirect injury (i.e., impacts that come to the lower level of the chin and cause the mandible to close with a large force against the maxillary).

In the primary dental system, displacement injuries are more common than fractures due to the nature of the spongy structure of bone in young children and the lower ratio of the root or the crown to the permanent teeth. Although reports of injuries in preschool children referred for dental treatment indicate that most of them suffer from displacement injuries. However, epidemiological studies in the community indicate that crown fractures are the most common damage in the primary teeth. However, these injuries can only cause a minor damage, unfortunately, this makes parents do not seek any dental treatment for their children. At age 5, about $40 \%$ of boys and $30 \%$ of girls experienced traumatic injuries to their teeth. At the age of 2-4, the amount of damage to the primary teeth is highest because, currently, the motor skills of the baby are evolving. Children with a protrusion in anterior incisors (evolving in class II malocclusions) 2-3 times more likely are prone to have dental damage than the children with normal incisors. In other words, the incisors teeth are more damaged during exercise, biking, running, etc. If the central incisors teeth are placed far anterior to the other teeth and there is a space with the other, or even both of the jaws are prognathous, the probability of injury and damage to the central incisors are much larger. Therefore, if the child has the prognathic jaws problems, parents should contact the surgeon or orthodontist as soon as possible to solve this problem.
To Submit Your Article Click Here:

This work is licensed under Creative Commons Attribution 4.0 License

DOI: 10.32474/IPDOAJ.2018.02.000134

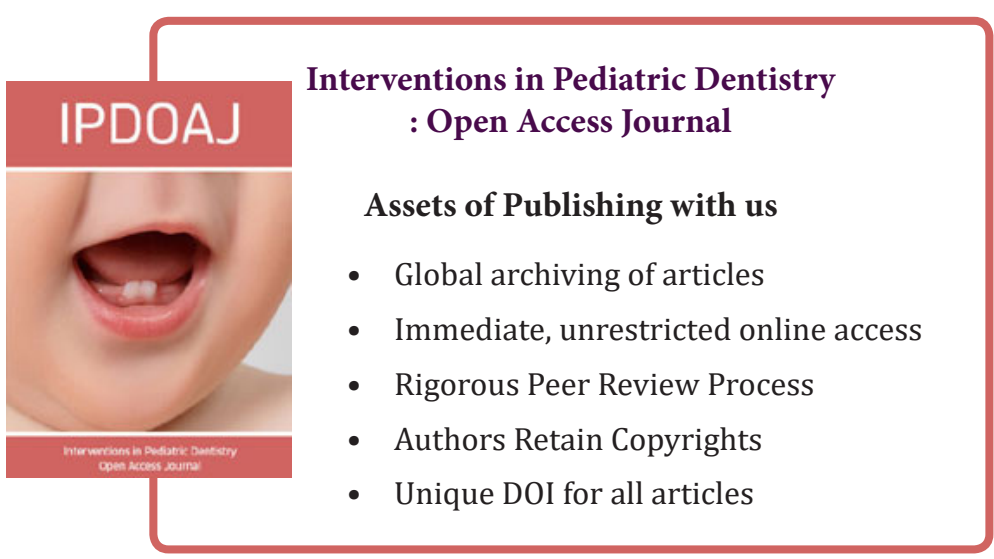

